\title{
A NATURAL LINEAR ORDERING OF BASIC COMMUTATORS
}

\author{
HERMANN V. WALDINGER
}

The basic commutators derived from the generators of a free group were introduced by Philip Hall and studied extensively by Marshall Hall, Jr. There exists a natural linear ordering for these commutators. It is the purpose of this paper to show that this ordering is, in a certain sense, invariant under "multiplication," i.e., under the process of forming the commutator with the same basic commutator on both sides of an inequality. We derive and state our results in the language of commutators in groups; obviously, they can be formulated also in terms of elements of a free Lie ring. Our investigation was motivated by a study of the smallest normal divisor in a free group containing a given basic commutator.

We start out by giving some notation and definitions which will be used throughout this paper.

$G$ will be the free group on $x_{1}, x_{2}, \cdots, x_{r}$. If $a, b \in G$ then $(a, b)$ $=a^{-1} b^{-1} a b$. The lower central series of $G$ is the chain $G_{1} \supset G_{2} \supset \ldots$ $G_{n} \supset G_{n+1} \supset \ldots$ of subgroups defined by setting $G_{1}=G, G_{n}=\left(G_{n-1}, G\right)$, the group generated by all commutators of the form $\left(a_{n-1}, b\right)$ with $a_{n-1} \in G_{n-1}$ and $b \in G$.

We wish now to construct basic commutators and at the same time define a linear ordering on them. This is done by induction. The basic commutators of weight one with their linear order are $x_{1}<x_{2}<\ldots$ $<x_{r}$. Having defined basic commutators of weight less than $n$ and ordered them, we use these to get the ones of weight $n$. The basic commutators of weight $n$ are $C_{n}=\left(C_{i}, C_{j}\right)$ where $C_{i}, C_{j}$ are basic commutators with weights $W\left(C_{i}\right)+W\left(C_{j}\right)=n, C_{i}>C_{j}$ and such that if $C_{i}=\left(C_{s}, C_{t}\right)$, then $C_{j} \geqq C_{t}$. Let $C_{n_{1}}=\left(C_{i_{1}}, C_{j_{1}}\right)$ and $C_{n_{2}}=\left(C_{i_{2}}, C_{j_{2}}\right)$ be of weight $n$. Then $C_{n_{1}}>C_{n_{2}}$ if $C_{i_{1}}>C_{i_{2}}$ or $C_{i_{1}}=C_{i_{2}}$ but $C_{j_{1}}>C_{j_{2}}$. A basic commutator of weight $n$ is greater than any of weight less than $n$. Thus, if $r=2$, let $x_{1}=x, x_{2}=y$ so that the basic commutators of weight $\leqq 3$ in their order are

$$
x<y<(y, x)<((y, x), x)<((y, x), y) .
$$

It should be noted that basic commutators of the same weight have been given a lexicographic ordering, unlike the usual definition of basic commutators which allows an arbitrary ordering of basic com-

Received by the editors March 24, 1960. 
mutators of the same weight [2]. The ordering in this paper will be preserved under commutation.

We shall assume the subscripts for the commutators chosen so that $C_{i}$ is the $i$ th basic commutator. It can be shown that a basic commutator of weight $n$ is in $G_{n}$ but not in $G_{n+1}$. Moreover, since the intersection of all terms of the lower central series is the identity, for any element $a$, there is a positive integer $K$ with $a \in G_{K}, a \notin G_{K+1}$. We call $K$ the weight $W(a)$ of $a$.

The following result is well known: if $a, b \in G$ and $(a, b) \notin G_{W(a)+W(b)+1}$ then $(a, b)$ can be written mod $G_{W(a)+W(b)+1}$ uniquely as a product of basic commutators $C_{i_{s}}$ whose weight is $W(a)+W(b)$, i.e.,

$$
(a, b)=\prod_{s=1}^{t} C_{i_{s}}^{e_{i_{s}}} \bmod G_{W(a)+W(b)+1} \cdots
$$

where $e_{i_{s}} \neq 0$.

We now wish to consider the preservation of order under commutation. If $a$ and $b$ are basic commutators, then $(a, b)$ need not be basic and we therefore can not apply our linear ordering to it. To do this we must associate some basic commutator with $(a, b)$. This will be done in a fashion such that if $(a, b)$ is basic, it is associated with itself. When $a, b(a \neq b)$ are both basic commutators, then $(a, b)$ $\in G_{W(a)+W(b)}$ and the following definition has meaning:

Definition. The maximal component of $(a, b)$, denoted $M(a, b)$ is $C_{i_{p}}{ }^{\prime}$ where $i_{p}=\max \left\{i_{1}, \cdots, i_{t}\right\}$, i.e., $C_{i_{p}}$ is the largest basic commutator occurring on the right hand side of (1).

We have now completed all preliminaries and are ready to state our main theorem.

TheOREM. Let $a, b, c$ be basic commutators such that

$$
b>c, \quad a \neq b, \quad a \neq c .
$$

Then

$$
M(a, b)>M(a, c) .
$$

The proof of this theorem will be accomplished easily once we have established the following auxiliary lemmas on the basic commutators $a, b, c$.

LEMma I. Let

$$
a>b>c
$$




$$
M(a, b)>M(a, c) .
$$

Lemma II. When (4) holds, then

$$
M(a, c)>M(b, c) .
$$

Lemma III. Suppose $a>b$ and

$$
M(a, b)=(\eta, \zeta)
$$

$$
a=(\alpha, \beta)
$$

then

$$
\zeta \leqq \beta \quad \text { or } \zeta \leqq b .
$$

On the other hand, if $W(a)=1$, then

$$
\zeta \leqq a \text { or } \zeta \leqq b
$$

We shall prove these lemmas simultaneously by induction on the number of basic commutators less than $a$. We note first that Lemma III holds trivially for $a=C_{2}, b=C_{1}$.

When $G$ has two generators only, we begin the induction with $a=C_{3}, W(a)=2$. We must take $b=C_{2}, c=C_{1}$. Then

$$
\begin{aligned}
M(a, b) & =\left(\left(C_{2}, C_{1}\right), C_{2}\right)>M(a, c)=\left(\left(C_{2}, C_{1}\right), C_{1}\right)>M(b, c) \\
& =\left(C_{2}, C_{1}\right) .
\end{aligned}
$$

But (11) verifies our lemmas for $a=C_{3}$.

When $G$ has more than two generators, however, we must start with $W(a)=1$. Let us take for $a$ the generator $C_{p_{1}}$. Let $b=C_{p_{2}}$, $c=C_{p_{3}}$ with $p_{1}>p_{2}>p_{3} \geqq 1$. Then

(12) $M(a, b)=\left(C_{p_{1}}, C_{p_{2}}\right)>M(a, c)=\left(C_{p_{1}}, C_{p_{8}}\right)>M(b, c)=\left(C_{p_{2}}, C_{p_{8}}\right)$.

And (12) shows that the three lemmas hold for $a=C_{p_{1}}$.

Let us now suppose that $W\left(C_{j}\right) \geqq 2$ and that we have proven our lemmas for every $a=C_{j}$ with $3 \leqq j<i$. We shall establish them below for $a=C_{i}$. We start out with Lemma IV which implies the validity of Lemma III for $a=C_{i}$.

Lemma IV. Suppose that Lemmas I, II, III hold for all basic commutators which are less than $a=C_{i}=(\alpha, \beta)$. Then the following formula holds for obtaining the maximal component of $(a, b)$, when $b<a$ :

$$
M(a, b)=\begin{array}{lll}
(a, b) & \text { when } & \beta \leqq b<a \\
(M(\alpha, b), \beta) & \text { when } & b<\beta<a .
\end{array}
$$


Proof. When $\beta \leqq b<a$, then $(a, b)=((\alpha, \beta), b)$ is a basic commutator and thus $M(a, b)=(a, b)$. For the more interesting case $b<\beta$ we take as point of departure the Jacobi identity

$$
((\alpha, \beta), b)((\beta, b), \alpha)((b, \alpha), \beta) \equiv 1 \bmod G_{W(\alpha)+W(\beta)+W(b)+1},
$$

which we shall write in the form:

$$
(a, b)=((\alpha, \beta), b) \equiv((\alpha, b), \beta)((\beta, b), \alpha)^{-1} \bmod G_{W(a)+W(b)+1} .
$$

Referring to (1) we have the unique expansions

$$
\begin{aligned}
& (\alpha, b) \equiv \prod_{s=1}^{m} C_{i_{s}}^{e_{s_{s}}} \bmod G_{W(\alpha)+W(b)+1} \\
& (\beta, b)=\prod_{t=1}^{n} C_{j_{t}}^{e_{j_{t}}} \bmod G_{W(\beta)+W(b)+1}
\end{aligned}
$$

Combining (15) and (16) we find that

$$
(a, b) \equiv \prod_{s=1}^{m} \prod_{t=1}^{n}\left(C_{i_{s}}, \beta\right)^{e_{i_{s}}}\left(C_{j_{t}}, \alpha\right)^{-e_{i t}} \bmod G_{W(a)+W(b)+1}
$$

Suppose that the basic commutators in (16) are ordered so that

$$
\begin{aligned}
i_{1} & =\max \left\{i_{1}, i_{2}, \cdots, i_{m}\right\}, \\
j_{1} & =\max \left\{j_{1}, j_{2}, \cdots, j_{n}\right\} .
\end{aligned}
$$

Then

$$
C_{i_{1}}=M(\alpha, b) .
$$

Since $\alpha<a=C_{i}$, we may apply Lemma I and find from $b<\beta<\alpha$

$$
C_{i_{1}}=M(\alpha, b)<M(\alpha, \beta)=a .
$$

Next we note that

$$
W\left(C_{i_{1}}\right)=W\left(C_{i_{s}}\right)>W(\alpha) \geqq W(\beta),
$$

and thus

$$
a>C_{i_{1}}>C_{i_{s}}>\alpha>\beta,
$$

for $s=2, \cdots, m$. We may apply Lemma II to yield

$$
M\left(C_{i_{1}}, \beta\right)>M\left(C_{i_{s}}, \beta\right) \text {. }
$$

Therefore

$$
M((\alpha, b), \beta)=M(M(\alpha, b), \beta)=M\left(C_{i_{1}}, \beta\right) .
$$


But Lemma III holds for $C_{i_{1}}<a$ by the induction hypothesis. It says that if $\alpha=(\gamma, \delta)$ and $C_{i_{1}}=(\eta, \zeta)$, then $\zeta \leqq b$ or $\zeta \leqq \delta$. Since $a=(\alpha, \beta)$ is basic, $\delta \leqq \beta$ and thus $\zeta \leqq \beta$ in any case. Hence, $\left(C_{i_{1}}, \beta\right)$ is a basic commutator and

$$
M\left(C_{\boldsymbol{i}_{1}}, \beta\right)=\left(C_{\boldsymbol{i}_{1}}, \beta\right) .
$$

Next we must examine $((\beta, b), \alpha)$. We shall consider three cases. First suppose that $W(\alpha)>W(\beta)+W(b)$. In this case

$$
\alpha>C_{j t}>\beta \quad \text { for } t=1,2, \cdots, n \text {. }
$$

It follows that every commutator $\left(\alpha, C_{j_{t}}\right)$ is a basic commutator of weight $W(\alpha)+W(b)$. But the basic commutator $\left(C_{i_{1}}, \beta\right)$ has the same weight and is greater than every $\left(\alpha, C_{j_{b}}\right)$ since $W\left(C_{i_{1}}\right)>W(\alpha)$ and thus $C_{i_{1}}>\alpha$. It is then clear from (17) that

$$
M(a, b)=\left(C_{i_{1}}, \beta\right) .
$$

The second case corresponds to $W(\alpha)=W(\beta)+W(b)$. But then all $\left(\alpha, C_{j_{t}}\right)$ are either basic commutators or inverses of such commutators. We note that $W(\alpha)=W\left(C_{j_{t}}\right)<W\left(C_{i_{1}}\right)$ and $W(\alpha)+W\left(C_{j_{t}}\right)=W\left(C_{i_{1}}\right)$ $+W(\beta)$ again implies $\left(\alpha, C_{j_{k}}\right)$ or $\left(C_{j_{k}}, \alpha\right)<\left(C_{i_{1}}, \beta\right)$ and so (27).

In the third case we make the hypothesis that $W(\alpha)<W(\beta)+W(b)$. We must first show that

$$
a=(\alpha, \beta)>M(\beta, b)=C_{j_{1}} .
$$

We note that

$$
a>\alpha>\beta>b .
$$

But (29) allows us to find from Lemma I that

$$
a=(\alpha, \beta)=M(\alpha, \beta)>M(\alpha, b),
$$

and from Lemma II that

$$
M(\alpha, b)>M(\beta, b) .
$$

Combining (30) and (31) we obtain (28).

Having established (28) we now may use Lemma II on $C_{j_{1}}>C_{j_{t}}>\alpha$ and see at once that

$$
M\left(C_{j_{1}}, \alpha\right)>M\left(C_{j_{t}}, \alpha\right) \quad \text { for } t=2, \cdots, n .
$$

Finally we note that $\beta<a$ and so by the induction hypothesis of Lemma III, if $M(\beta, b)=C_{j_{1}}=(\lambda, \mu)$ then $\mu<\beta, b$ and so $\left(C_{j_{1}}, \alpha\right)$ is a basic commutator. This means that

$$
M\left(C_{j_{1}}, \alpha\right)=\left(C_{j_{1}}, \alpha\right) .
$$


But $W\left(C_{j_{1}}, \alpha\right)=W\left(C_{i_{1}}, \beta\right)$ and $C_{i_{1}}=M(\alpha, b)>C_{j_{1}}=M(\beta, b)$, since we know Lemma II to hold by virtue of (29). We again arrive at (27) and have now proven under the inductive hypothesis of Lemmas I, II and III that Lemma IV and hence its consequent Lemma III hold.

Next we deduce the validity of Lemma I for $a=C_{i}$ from Lemma IV. To do so we examine the sequences

$$
\begin{aligned}
M\left(a, C_{i-1}\right), M\left(a, C_{i-2}\right), \cdots, & M\left(a, C_{v}\right) \\
& =M(a, \beta), M\left(a, C_{v-1}\right), \cdots M\left(a, C_{1}\right),
\end{aligned}
$$

where we write $C_{v}$ for the basic commutator $\beta$. Because of Lemma IV, it is identical with

$$
\begin{aligned}
\left(a, C_{i-1}\right), & \left(a, C_{i-2}\right), \cdots,\left(a, C_{v}\right) \\
& =\left(\left(\alpha, C_{v}\right), C_{v}\right),\left(M\left(\alpha, C_{v-1}\right), C_{v}\right), \cdots,\left(M\left(\alpha, C_{1}\right), C_{v}\right) .
\end{aligned}
$$

For Lemma I, it is evidently sufficient to show that

$$
\left(a, C_{\mu+1}\right)>\left(a, C_{\mu}\right) \quad \text { for } v \leqq \mu<i-1,
$$

and also that

$$
\left(M\left(\alpha, C_{\nu+1}\right), C_{v}\right)>\left(M\left(\alpha, C_{v}\right), C_{v}\right) \quad \text { for } 1 \leqq \nu \leqq v-1 .
$$

Since $W\left(C_{\mu+1}\right) \geqq W\left(C_{\mu}\right), W\left(a, C_{\mu+1}\right) \geqq W\left(a, C_{\mu}\right)$ and hence by the definition of the linear ordering (36) follows. Since $a>\alpha>\beta \geqq C_{v+1}$ $>C_{v}$, we can apply our inductive hypothesis on Lemma I to obtain $M\left(\alpha, C_{\nu+1}\right)>M\left(\alpha, C_{\nu}\right)$; hence, again by the definition of the linear ordering (37) follows.

We have demonstrated Lemmas I and III for $a=C_{i}$. It remains to examine Lemma II. When $W(a)>W(b)$, then $W(M(a, c))$ $>W(M(b, c))$ and therefore

$$
M(a, c)>M(b, c) .
$$

It is thus sufficient to proceed under the hypothesis $W(a)=W(b) \geqq 2$ from here on. We shall write

$$
\begin{aligned}
& a=\left(\alpha_{1}, \beta_{1}\right), \\
& b=\left(\alpha_{2}, \beta_{2}\right),
\end{aligned}
$$

and consider the four cases:

$$
\begin{array}{ll}
\text { 1. } \beta_{1} \leqq c, & \beta_{2} \leqq c \\
\text { 2. } c<\beta_{1}, & \beta_{2} \leqq c \\
\text { 3. } \beta_{1} \leqq c, & c<\beta_{2} \\
\text { 4. } c<\beta_{1}, & c<\beta_{2} .
\end{array}
$$


1. $(a, c)=M(a, c)$ and $(b, c)=M(b, c)$ are basic commutators. Since $a>b$ and $W(a, c)=W(b, c),(a, c)>(b, c)$ by definition.

2. From Lemma IV we find that

$$
\begin{aligned}
& M(a, c)=\left(M\left(\alpha_{1}, c\right), \beta_{1}\right), \\
& M(b, c)=\left(\left(\alpha_{2}, \beta_{2}\right), c\right) .
\end{aligned}
$$

We note that

$$
\beta_{2} \leqq c<\beta_{1} .
$$

Since $a>\alpha_{1}$ we already know from Lemma I that

$$
M\left(\alpha_{1}, c\right) \geqq M\left(\alpha_{1}, \beta_{2}\right) .
$$

But $a>b$ and $W(a)=W(b)$, hence $\alpha_{1} \geqq \alpha_{2}$ by definition. Thus,

$$
M\left(\alpha_{1}, \beta_{2}\right) \geqq M\left(\alpha_{2}, \beta_{2}\right)=\left(\alpha_{2}, \beta_{2}\right)
$$

by the inductive hypothesis for Lemma II. Combining the above we find

$$
M\left(\alpha_{1}, c\right) \geqq\left(\alpha_{2}, \beta_{2}\right) .
$$

Since

$$
W\left(M\left(\left(\alpha_{1}, c\right), \beta_{1}\right)\right)=W(M(a, c))=W(M(b, c))=W\left(\left(\alpha_{2}, \beta_{2}\right), c\right),
$$

we find from (44) and (41) and the definition of the ordering, that

$$
\left(M\left(\alpha_{1}, c\right), \beta_{1}\right)>\left(\left(\alpha_{2}, \beta_{2}\right), c\right) .
$$

3.

$$
\begin{aligned}
& M(a, c)=\left(\left(\alpha_{1}, \beta_{1}\right), c\right), \\
& M(b, c)=\left(M\left(\alpha_{2}, c\right), \beta_{2}\right)
\end{aligned}
$$

by Lemma IV. We note that $a>\alpha_{1} \geqq \alpha_{2}>\beta_{2}>c$ since $a>b$. Hence by the inductive hypothesis for Lemma I

$$
b=\left(\alpha_{2}, \beta_{2}\right)=M\left(\alpha_{2}, \beta_{2}\right)>M\left(\alpha_{2}, c\right),
$$

and so

$$
a=\left(\alpha_{1}, \beta_{1}\right)>b=\left(\alpha_{2}, \beta_{2}\right)>M\left(\alpha_{2}, c\right) .
$$

Furthermore

$$
\begin{aligned}
& W\left(\left(\alpha_{1}, \beta_{1}\right), c\right)=W\left(\alpha_{1}\right)+W\left(\beta_{1}\right)+W(c) \\
& \geqq W\left(\alpha_{2}\right)+W\left(\beta_{1}\right)+W(c) \\
&=W\left(M\left(\alpha_{2}, c\right), \beta_{1}\right)
\end{aligned}
$$


since $\alpha_{1} \geqq \alpha_{2}$. But (48) and (49) imply that

$$
\left(\left(\alpha_{1}, \beta_{1}\right), c\right)>\left(M\left(\alpha_{2}, c\right), \beta_{1}\right)
$$

by the definition of the ordering.

4.

$$
\begin{aligned}
& M(a, c)=\left(M\left(\alpha_{1}, c\right), \beta_{1}\right), \\
& M(b, c)=\left(M\left(\alpha_{2}, c\right), \beta_{2}\right)
\end{aligned}
$$

by Lemma IV. Since $a>\alpha_{1} \geqq \alpha_{2}$ we may apply Lemma II to yield

$$
M\left(\alpha_{1}, c\right) \geqq M\left(\alpha_{2}, c\right)
$$

the equality holding only for $\alpha_{1}=\alpha_{2}$. But when $\alpha_{1}=\alpha_{2}$, then $\beta_{1}>\beta_{2}$ since $a>b$. Since $W(a)=W(b)$,

$$
M(a, c)>M(b, c)
$$

under both circumstances by the definition of the ordering.

This concludes our examination of Lemma II. We have now shown that the hypothesis that Lemmas I, II and III hold for every $a=C_{j}$ with $3 \leqq j<1$ means that they must also hold for $a=C_{i}$. The lemmas have thus been established by induction.

We are now ready to prove the theorem. Since $b>c$ and $a \neq b, a \neq c$ there are three possible cases:

$$
a>b>c .
$$

The theorem is identical with Lemma I.

$$
b>a>c \text {. }
$$

Then $M(a, b)=M(b, a)>M(b, c)$ by Lemma I. Also $M(b, c)>M(a, c)$ by Lemma II. Hence $M(a, b)>M(a, c)$.

$$
b>c>a \text {. }
$$

The theorem is identical with Lemma II.

\section{BIBLIOGRAPHY}

1. Philip Hall, $A$ contribution to the theory of groups of prime power order, Proc. London Math. Soc. vol. 36 (1933) pp. 29-95. 1959.

2. Marshall Hall, Jr., The theory of groups, New York, The Macmillan Company,

Republic Aviation Corporation 